\title{
Grammatical frames as stimuli in a four-stage, stimulus-equivalence paradigm
}

\author{
David T. Hakes, Carlton T. James' and Bobert H. Lloyd \\ UNIVERSITY OF TEXAS
}

\begin{abstract}
Abstraet
The four-stage, stimulus-equivalence paradigm was studied using sentence frames as stimuli and low meaningfulness paralogs as responses. The first three lists were presented as self-paced PA tasks, and the fourth, or test, list was presented as a matching task. In accord with the results of a previous experiment, highly significant mediated transfer was obtained. Unlike the earlier experiment, however, there was no evidence that mediation occurred as an all-or none phenomenon for all pairs in the test list. The difference in results was attributed to slower learning and poorer retention of the materials used in the present experiment.
\end{abstract}

\section{Problem}

Numerous experimenters (cf., Jenkins, 1961) have obtained mediated transfer in three-stage paradigms. Considerably greater difficulty has been experienced in obtaining comparable results in four-stage paradigms. Using experimental designs in which two of the four stages were inferred from word association norms, Russell \& Storms (1955) and McGeehee \& Schulz (1961) found evidence of mediated transfer in a four-stage chaining paradigm. Such transfer has been more difficult to obtain, however, when the attempt is made to have Ss acquire all four stages in the laboratory.

James \& Hakes (1965) have recently demonstrated mediated transfer in a laboratory-acquired, four-stage paradigm. Using self-paced PA tasks for the first three stages of a stimulus-equivalence paradigm and a matching task for the fourth, or test, stage, strong evidence of mediation was obtained. In addition, their results appeared to indicate that Ss either mediated on all test-list pairs or not at all. Further, it appeared that test-list performance was perfectly correlated with S's ability to report mediating.

The present experiment was designed to determine if the James \& Hakes findings were replicable, both in general and with respect to the apparent all-or-none character of mediation. For this reason, the procedures of the earlier experiment were repeated using different classes of verbal material.

\section{Method}

Two basic conditions, Experimental and Control, were included. The four lists for the Experimental condition formed an $A-B, C-B, A-D, C-D$ paradigm. For the Control condition, an $\mathrm{X}-\mathrm{D}$ list was substituted for the A-D list. Two sets of lists, formed from different sets of items, were used. A total of 48 Ss served in the experiment and were assigned to conditions according to a random sequence.
Jenkins (1964) has suggested that the stimulusequivalence paradigm is one in which both associative and grammatical aspects of words are acquired. To make the experimental situation somewhat comparable to the language acquisition situation, the stimuli used were sentence frames. Twenty-four simple sentences were composed, and from each the subject was deleted. These sentence frames served as the A and C terms, and the $B$ and $D$ terms (i.e., responses) were paralogs selected for low meaningfulness from Taylor's (1959) norms. Thus, in each stage, Ss were to learn which of six paralogs was appropriate in the context of each of six sentence frames.

Both sentence frames and paralogs were randomly divided into two sets of 12 items each. The 12 frames and paralogs of each set were combined to yield a set of four lists. For the Control condition, the $\mathrm{X}$ items of one set of lists were the A items from the other set. The first three lists were prepared on $3 \times 5$ cards, with the sentence frame on one side and the frame with the appropriate paralog inserted and underscored on the other. The test list contained a listing of the $\mathrm{C}$ frames and a separate listing of the D paralogs. Four random orders of the $\mathrm{C}$ and $\mathrm{D}$ items were prepared for each item set.

For the first three lists, the first trial involved presenting each stimulus alone for 2 sec., followed by the S-R pair for $8 \mathrm{sec}$. On subsequent trials, each stimulus was presented until Ss responded, following which the S-R pair was presented for $2 \mathrm{sec}$. The cards were shuffled after each trial. Learning on each list was carried to a criterion of two successive errorless trials. Second-list learning was followed by two recallpractice trials on the first list, and third-list learning was followed by two recall-practice trials on each of the three lists.

Ss were then presented the test-list matching task and instructed to match each paralog with a sentence frame. The experiment concluded by asking $S$ to describe how he had arrived at his test-list matches.

\section{Results}

Trials to reach a criterion of two successive errorless trials were analyzed for each of the three PA learning tasks. In the analyses of first-and second-list learning, Conditions, Lists, and the interaction all failed to reach significance, providing support for the hypotheses of equivalent groups and lists. The analysis of third-list learning yielded no significant main effects. The Conditions $\mathrm{x}$ Lists interaction was, however, significant, $F(1,44)=4.78 ; p<.05$. 
Mean numbers of correct responses on the first trial of each of the recall-practice tasks were also analyzed. Analysis of the first A-B recall (i.e., after C-B learning) yielded no significant effects. The second A-B recall task yielded a significant effect for Conditions, $F(1,44)=5.09 ; p<.05$, no other effect approaching significance. Mean numbers of correct responses were 5.17 and 5.71 for the Experimental and Control conditions respectively, indicating retroactive interference for the Experimental condition, a finding comparable to that obtained by James \& Hakes.

Analysis of C-B recall failed to yield any significant effects. Recall of the third list (A-D or X-D) showed a significant Conditions effect, $F(1,44)=4.70 ; p<.05$. The Experimental and Control conditions yielded means of 5.25 and 5.71 correct responses, a finding also comparable to that obtained in the earlier experiment.

On the test list, the Experimental and Control groups matched 3.79 and 0.67 correct pairs respectively. Conditions was the only significant effect in the analysis, $F(1,44)=43.30 ; p<.001$. Whereas the previous experiment indicated that Experimental Ss either matched perfectly or performed at chance, the present experiment showed a more continuous distribution of performance between chance and perfect. In addition, the post-experimental inquiry failed to reveal a relationship between test-list performance and awareness of mediation.

\section{Diseussion}

The present results are generally supportive of those obtained previously. The lack of differences on any of the learning tasks, the differences in recall, and the test-list difference are all highly similar. It would appear that the mediation effect obtained using these procedures is both large and stable.

The major difference between the present and previous results is in the distribution of correct responses on the test list. The all-or-none effect previously noted was not replicated. Similarly, the results of the postexperimental inquiry did not permit classifying Experi- mental Ss into "mediators" and "nonmediators." Since only the materials differed between the experiments, it would seem that the difference in results is attributable to this difference.

In the James \& Hakes experiment, all items were highly meaningful nouns. The results of the recall tasks indicated that no $S$ failed to recall any pair on the second trial on any list. The more difficult materials used in the present experiment might be expected to result in slower learning and poorer retention. With the exception of the C-B list, all lists in the present experiment were learned more slowly than the comparable lists in the previous experiment. In addition, a number of Ss failed to recall the lists perfectly on the second recall trials. This would suggest that failure to mediate perfectly on the test list is in part attributable to inability to recall the associations from the previous lists.

Although learning was more difficult and retention poorer in the present experiment, the overall amount of mediated transfer was, if anything, greater. It would appear, therefore, that mediated transfer does occur in the paradigm tested and is not greatly affected by the materials used. The apparent all-or-none effects appear to be more dependent upon the materials, probably due to differences in retention.

\section{References}

JAMES, C. T., \& HAKES, D. T. Mediated transfer in a four-stage, stimulus-equivalence paradigm. J. verbal. Learn. verbal Behav., 1965, in press.

JENKINS, J. J. Mediated associations: Paradigms and situations. In C. N. Cofer \& B. S. Musgrave (Eds.), Verbal behavior and learning: Problems and processes. New York: McGraw-Hill, 1963. JENKINS, J. J. A mediational account of grammatical phenomena. J. Communication, 1964, 14, 86-97.

MCGEEHEE, N. E., \& SCHULZ, R.W. Mediation in paired-associate learning. J. exp. Psychol., 1961, 62, 565-570.

RUSSELL, W. A., \& STORMS, L. H. Implicit verbal chaining in paired-associate learning. J. exp. Psychol., 1955, 49, 267-293.

TAYLOR, J. D. The meaningfulness of 320 words and paralogs. Unpublished doctoral dissertation, Duke University, 1959.

Note

1. Now at Indiana University. 